\title{
Structurations formelle et conceptuelle des articles de dictionnaires : le premier modèle de codification des articles dans l'histoire des dictionnaires Larousse
}

\author{
Corbin, Pierre \& Gasiglia, Nathalie \\ UMR 8163 (STL) du CNRS, Université Lille 3 \\ pierre.corbin@univ-lille3.fr \& nathalie.gasiglia@univ-lille3.fr
}

L'intertextualité est inhérente à l'activité lexicographique. La glossographie et la lexicographie des copistes médiévaux ont produit de nombreuses variantes évolutives de diverses matrices dictionnairiques, échelonnées sur plusieurs siècles. ${ }^{1}$ Dans les débuts de l'imprimerie commerciale, Robert Estienne, puisant lui-même dans cette tradition, procéda par inversion de nomenclature et ajustements textuels pour décliner ses dictionnaires bilingues latin / français en les adaptant à différents niveaux de compétence. ${ }^{2}$ Et de nos jours, les éditeurs spécialisés combinent et modulent, selon les besoins des différents jalons de leurs catalogues, les données stockées dans leurs bases de ressources. Dans la période contemporaine, la recherche métalexicographique a fait un sort à cette intertextualité, qu'elle soit envisagée au titre de la "criminalité dictionnairique", , comme dans le plagiat ${ }^{4}$ ou d'autres pratiques éditoriales discutables ${ }^{5}$, ou à celui de l' « histoire interne $»^{6}$ de certains dictionnaires, pour établir des généalogies textuelles ${ }^{7}$, évaluer la portée de refontes ou de mises à jour ${ }^{8}$, dessiner des filières dans l'évolution de la production de maisons d'édition ${ }^{9}$, disséquer des démarches de dérivation rédactionnelle ${ }^{10}$ ou pour d'autres visées encore.

Si un corpus non négligeable d'études de filiations textuelles entre dictionnaires s'est ainsi progressivement constitué, il n'en va pas de même pour les codifications mises en œuvre dans les ouvrages pour discriminer, hiérarchiser et ordonner les informations, dont l'analyse n'a pas été dynamisée par un texte de cadrage documenté paru il y a maintenant un quart de siècle ${ }^{11}$. Ces dispositifs sémiotiques sont pourtant de première importance, puisqu'ils déterminent la fonctionnalité des répertoires en guidant leurs utilisateurs vers leur compréhension, tout en révélant les moyens techniques mobilisables et en reflétant des esthétiques en vigueur pour diverses catégories de produits à des époques déterminées. Cette observation nous a incités à engager des recherches sur ce terrain dans diverses directions. L'objet de cette contribution est d'en présenter certains résultats en décrivant une partie de l'évolution des codes utilisés dans les articles des dictionnaires Larousse depuis la création de l'entreprise au milieu du XIX ${ }^{\mathrm{e}}$ siècle.

L'examen de ces codes permet trois ordres de considérations :

(i) Ils puisent dans les quatre ensembles de moyens exploitables ${ }^{12}$ :

- les moyens textuels, avec d'une part l'utilisation d'abréviations pour la catégorisation des unités lexicales, repère essentiel de l'organisation des articles, et pour les marques (de domaines, de registres, d'usualité ou sémantiques), qui sont d'autres indicateurs de leur découpage, et d'autre part la mutualisation de certains segments textuels ;

- les moyens graphématiques, avec l'association des oppositions entre majuscules et minuscules à l'initiale de séquences graphiques définies et des ponctuations, utilisées stratégiquement pour relier (virgule, deux points) ou pour séparer (point, point-virgule), selon les visées ;

- les moyens symboliques, avec l'emploi d'éléments diacritiques (tirets, losanges, barres verticales...) et alphanumériques ;

- et les moyens typographiques, avec des contrastes affectant les caractères (romain vs italique, capitales $v s$ bas de casse, variation des degrés de graisse...) et des choix de mise en page (articles en texte continu ou fractionnés en paragraphes mis en évidence par des retraits et, parfois, des interlignes variables).

(ii) Ils varient en fonction des types de dictionnaires concernés : les contrastes observables entre deux ouvrages de même concepteur et proches dans le temps, comme le furent, par exemple, le Nouveau dic- 
tionnaire de la langue française [NDLF] (1856) et le Grand dictionnaire universel du XIX siècle [GDU] (1866-1876), dus à Pierre Larousse, permettent d'imputer à leurs visées et à leurs programmes différents les contrastes formels qu'ils présentent (cf. figures 1 et 22), qu'il s'agisse des options de mise en page (texte d'un seul bloc $v s$ découpage en paragraphes à retrait de première ligne introduits par des tirets et précédés d'un interligne particulier) ou du choix de ne pas mettre en relief l'item vedette dans les contextualisations ou au contraire de lui affecter une typographie spécifique (petites capitales romaines dans un contexte de bas de casse italiques, ou italique dans un contexte en romain, selon les composants d'articles impliqués (cf. § 5.1)).

(iii) Ils sont utilisés, quels que soient les types de répertoires et les époques, avec une sobriété qui évite le surcodage des différenciations formelles servant à l'identification des composants des articles, au risque même d'insuffisances de discrimination.

La suite de notre propos illustrera ces points en faisant apparaître des jalons dans l'histoire de l'articulation entre la structure conceptuelle et la structure formelle des articles ${ }^{13}$ des dictionnaires généraux monolingues Larousse imprimés des origines à nos jours. Au regard de l'ampleur des données à couvrir, il ne s'agira que d'un survol, qui permettra cependant

- de présenter le premier des trois modèles majeurs de codification des articles dans l'histoire de ces répertoires, qui trouve son origine dans le $N D L F$ de 1856 ( $(1)$ ou dans l'une de ses éditions révisées dans les décennies suivantes $(\S 2)$,

- d'inventorier et de typer la longue postérité du modèle de 1856 et de sa forme révisée dans l'histoire des dictionnaires Larousse ( $\S \S 3$ et 4$)$,

- et de donner un aperçu, contrastivement au premier, des deux autres modèles majeurs, qui remontent respectivement au $G D U$ et au Dictionnaire du français contemporain $[D F C]$ de 1966, dont les codifications furent notamment caractérisées par l'introduction de symboles, diacritiques dans le premier et alphanumériques dans le second $(\S 5){ }^{14}$

Le traitement des items dont le lemme est $d r u$ servira de fil conducteur, mais d'autres données contribueront à enrichir les analyses.

\section{Le modèle du Nouveau dictionnaire de la langue française de $\mathbf{1 8 5 6}$}

Le premier modèle de structuration des articles laroussiens, celui du $N D L F$, monovolume de format assez petit $^{15}$ pour le grand public publié par Pierre Larousse en 1856, a eu pour caractéristique remarquable de ne pas utiliser significativement de symboles comme repères structurels. Ce modèle et ses évolutions ont perduré longtemps au sein du catalogue Larousse (cf. $\S \S 3$ et 4).

Le contenu textuel de l'article consacré aux unités lexicales dont le lemme est $d r u$ dans le $N D L F$ de 1856 est reproduit avec sa codification dans la figure 1:

Figure 1 - Article dru du Nouveau dictionnaire de la langue française de 1856 ( $3^{\mathrm{e}}$ édition)

DRU, E, adj. Se dit des petits oiseaux qui sont prêts à quitter le nid. Fig. Épais, serré, touffu : blés fort drus ; pluie drue et fine; gaillard, vif, gai : vous êtes bien dru aujourd'hui. Adv. En grande quantité, serré : tomber, semer dru.

Cette codification se caractérisait par l'économie des moyens mis en œuvre, puisqu'elle ne recourait ni à ceux, typographiques, de la mise en page (le retrait de première ligne servant au repérage des articles mais ne contribuant pas à la matérialisation de leur structure), ni aux symboles (à l'exception de quelques tirets sporadiques sans rôle régulier - comme s.v. alerte avant des recatégorisations ou s.v. amour pour des sens -, et d'un guillemet précédant les mots en adresse commençant par la lettre $h$ et soumis au hiatus, qui n'a pas de rôle dans la structuration des articles - comme s.v. hasard, cf. figure 3). Seuls étaient utilisés les moyens textuels (sous la forme des divers types de réductions qui concernent des formes ou des occurrences d'un item dont traite l'article ou qui affectent des éléments métalinguistiques qui introduisent des 
subdivisions de celui-ci), les moyens typographiques des variations de caractères (gras, italique, capitales) et les moyens graphématiques (oppositions entre majuscules et minuscules, ponctuations).

\subsection{Potentiel du dispositif de codification}

Il en allait de même dans tout le dictionnaire, y compris pour des articles plus complexes, que ce soit

- par le nombre d'occurrences des composants observés dans l'article dru, ce qui était le cas, par exemple, dans l'article clair, qui, comme le montre la figure 2, distribuait sur les trois catégories « adj. », «N. m. » et « Adv. » douze définitions, dont onze étaient illustrées par une contextualisation, et qui, pour deux d'entre elles, correspondaient à des sens figurés de l'adjectif, le tout étant articulé par le jeu sans faille des codifications graphématiques et des variations de caractères :

Figure 2 - Article clair du Nouveau dictionnaire de la langue française de 1856 ( $3^{\mathrm{e}}$ édition)

CLAIR, E, adj. Lumineux, éclatant ; qui reçoit beaucoup de jour : chambre claire ; poli, luisant : armes claires; transparent : eau claire; peu foncé en couleur : rose-clair ; peu consistant : sirop clair ; peu serré : toile claire ; pur, serein : temps clair. Fig. Intelligible : style clair ; évident, manifeste : preuve claire. N. m. Clarté : le clair de lune. Adv. D’une manière claire, distincte : voir clair.

- ou en raison de la présence de composants absents de l'article dru, comme des séquences lexicales complexes articulées aux gloses qui les suivent par une virgule et codées, pour certaines, en italique maigre et, pour d'autres, en romain gras (selon des critères de répartition qui ne seront pas analysés ici), dont l'article hasard, reproduit dans la figure 3, fournit des occurrences :

Figure 3 - Article hasard du Nouveau dictionnaire de la langue française de 1856 ( $3^{\mathrm{e}}$ édition)

"HASARD, n. m. Fortune, sort ; cas fortuit. Pl. Fig. Risques, périls : les hasards de la guerre. Jeu de hasard, où le hasard seul décide. Loc. adv. Au hasard, à l'aventure ; à tout hasard, quoi qu'il arrive ; par hasard, fortuitement.

Utilisé avec régularité, le protocole de mise en forme des articles du NDLF de 1856 était assez puissant pour permettre dans beaucoup de cas à des lecteurs attentifs de discerner leur organisation conceptuelle. Comme on a pu l'entrevoir dans la figure 2 à propos de l'article clair, l'aisance à percevoir celle-ci pouvait être inversement proportionnelle à la complexité des développements, mais le nombre élevé des articles brefs facilitait la tâche des utilisateurs.

\subsection{Limites du dispositif de codification}

Dans divers cas, cependant, de possibles doutes sur la compréhension de l'organisation des articles manifestent les limites du dispositif adopté. Trois exemples impliquant des séquences lexicales complexes illustrent différents cas de figure :

(i) Dans l'article hasard (figure 3), l'articulation majeure est la distinction catégorielle entre ce qui concerne le substantif («n. m. ») et ce qui relève des locutions adverbiales («Loc. adv.»), regroupées à la fin. Vient ensuite, pour le nom, la distinction entre ses emplois de lexème simple et celui dans lequel il est un élément de l'expression «Jeu de hasard», glosée mais non catégorisée comme telle. Enfin, pour le nom employé seul, il faut encore distinguer entre l'utilisation figurée au pluriel («Pl. Fig. ») et celle, sans restrictions, qui la précède. Sans être indécelable, cette structure conceptuelle ne se laisse sans doute pas aussi aisément détecter que si elle était signalée par des moyens symboliques et/ou de mise en page.

(ii) Comme on peut le voir dans l'article cas (figure 4), les séquences lexicales complexes codées en caractères romains gras qui sont catégorisées comme locutions pouvaient être réunies à une place non finale :

Figure 4 - Article cas du Nouveau dictionnaire de la langue française de 1856 ( $3^{\mathrm{e}}$ édition)

CAS, $n$. m. Evénement fortuit : le cas est extraordinaire; circonstance, conjoncture : que faire en pareil cas? Faire cas, estimer. Loc. adv. En ce cas, alors; en tout cas, quoi qu'il arrive. Gram. Désinence des mots : les six cas de la langue latine. 
Ici, c'est l'opposition entre l'usage terminologique dans le domaine grammatical (« Gram. ») et les autres emplois du mot, traités au préalable, qui constitue l'articulation première de l'article, et c'est au titre de ces emplois non spécialisés qu'il faut distinguer ce qui concerne le lexème simple, l'expression glosée non catégorisée « Faire cas 》, et les locutions adverbiales ( Loc. adv.») «En ce cas » et « en tout cas », dont la codification catégorielle est ici en italique alors qu'elle était en romain dans l'article hasard, ce qui reste à interpréter : irrégularité ou différenciation typographique délibérée ?

(iii) Un dernier article, droit (figure 5), montre que les séquences lexicales complexes en romain gras n'étaient pas nécessairement regroupées, ni catégorisées, ce qui induit une nouvelle nécessité d'adaptation pour l'interprétation structurelle du texte :

Figure 5 - Article droit du Nouveau dictionnaire de la langue française de 1856 ( $3^{\mathrm{e}}$ édition)

DROIT, n. m. Faculté de faire un acte, de jouir d'une chose, d'en disposer, ou d'exiger quelque chose d'une autre personne ; jurisprudence, législation : étudier le droit; impôt, taxe : droit d'enregistrement; justice : faire droit. Droit divin, qui vient de Dieu. Pl. Imposition : les droits d'entrée. A bon droit, loc. adv. Avec raison.

L'articulation principale sépare ici les développements sur le nom masculin («n. m. ») et sur la locution adverbiale « A bon droit », que suit la catégorisation « loc. adv. », alors qu'elle était antéposée et mise en facteur commun aux locutions regroupées des articles hasard et cas. Pour le nom, ce qui concerne le pluriel seul ( «Pl. ») contraste ensuite avec le développement précédent, qui porte sur ce qui n'est pas soumis à cette restriction, et c'est au sein de celui-ci qu'opère la distinction entre ce qui a trait au lexème simple et l'expression « Droit divin », codée comme une locution mais non catégorisée.

\section{2 Évolutions du modèle du Nouveau dictionnaire de la langue française}

Assez vite, à une date pas postérieure à 1878 d'après les exemplaires consultés ${ }^{16}$, le dispositif de codification du $N D L F$ s'est enrichi du recours récurrent à un symbole diacritique, le tiret, pour séparer le texte préexistant de certains articles de remarques linguistiques introduites à la fin de ceux-ci (qui concernaient la conjugaison, l'orthographe, l'usage grammatical...), comme dans l'article malgré d'une édition de 1878 reproduite dans la figure 6, dans laquelle la séquence ajoutée au texte de 1856 est surlignée (et qui permet d'observer que, dans cette édition, la catégorie n'est plus séparée de l'adresse par une virgule) :

Figure 6 - Article malgré du Nouveau dictionnaire de la langue française de 1878 (43 édition)

MALGRÉ prép. Contre le gré de ; nonobstant : malgré la pluie. - On ne dit plus malgré que dans le sens de quoique.

Ensuite, pas après 1886, le même tiret a été utilisé comme séparateur, dans certains articles, entre leur texte métalinguistique et des ajouts culturels redistribués, éventuellement avec des modifications, à partir d'articles indépendants qui étaient regroupés antérieurement, sous l'intitulé « Notes étymologiques, scientifiques, historiques et littéraires ", dans une deuxième nomenclature des dictionnaires ${ }^{17}$. L'article chlore d'une édition de 1886, reproduit dans la figure 7 avec surlignage de l'ajout, illustre cette disposition :

Figure 7 - Article chlore du Nouveau dictionnaire de la langue française de 1886 (66 édition)

CHLORE n. m. (gr. chlôros, jaune verdâtre). Corps simple, gazeux, d'une odeur forte et suffocante. - Gazeux ou dissous dans l'eau, le chlore, par son affinité pour l'hydrogène, détruit la partie colorante des matières végétales et animales. C'est à cause de cette propriété que l'industrie l'emploie pour le blanchiment des tissus. On s'en sert aussi pour détruire les miasmes délétères répandus dans l'atmosphère. On l'utilise souvent dans les cimetières, dans les hôpitaux, et surtout les salles de dissection.

Selon leur contenu textuel, ces ajouts culturels pouvaient comporter, comme dans l'article pétrification (figure 8), des passages à la ligne avec retrait de première ligne, qui constituaient un recours à des moyens typographiques de mise en page qui n'étaient pas utilisés par ailleurs : 
Figure 8 - Article pétrification du Nouveau dictionnaire de la langue française de 1886 (66 édition)

PÉTRIFICATION n. f. (lat. petra, pierre; facere, faire). Changement en pierre d'un corps organisé ; la chose pétrifiée : belle pétrification. - A la place de la substance dont il était composé, le corps pétrifié offre une matière étrangère et inorganique, représentant fidèlement sa structure. Les molécules du corps organique, détruites par le temps, sont remplacées par des molécules minérales qui prennent la même forme et occupent la même place. On comprend ainsi que la pétrification ait la même structure que le corps primitif.

Les pétrifications calcaires ou artificielles sont celles que l'on obtient en exposant des végétaux, des animaux, etc., à des sources renfermant une dissolution de carbonate de chaux. Mais ici la pétrification n'est qu'apparente et ne consiste, à proprement parler, que dans l'enveloppe ; le corps n'a pas changé de nature.

\section{Postérité du modèle de 1856}

Le modèle du NDLF initial de 1856 s'est propagé dans plusieurs dictionnaires Larousse de petit format utilitaires ou didactiques -, dont les principaux jalons ont été, chronologiquement et pour des durées déterminées, le Petit dictionnaire français, Le plus petit Larousse, le Dictionnaire des débutants et le Larousse de poche (tous illustrés ci-dessous par leur article dru non commenté) :

\subsection{Le Petit dictionnaire français de 1936 à 1978 / 1988}

Le premier dictionnaire de type nouveau à avoir repris (avec des variations typographiques : première ligne en débord, adresse en caractères bâton) les principes de codification structurelle du NDLF de 1856 fut le Petit dictionnaire français, ouvrage de format réduit ${ }^{18}$ publié en 1936 par Paul Augé sans indication de public destinataire (figure 9):

Figure 9 - Article dru du Petit dictionnaire français de 1936 (tirage de 1941) dru, e adj. Fort, vigoureux. Serré : pluie drue. Gaillard, vif. Adv. En grande quantité : tomber
dru.

Ces principes ont été conservés jusqu'à l'édition refondue de 1978 (recomposée en 1988) incluse, seul le texte des articles présentant des variations concernant le traitement de l'adjectif : "adj. Fort, vigoureux. » dans l'édition de 1956, « adj. Epais, serré, touffu. » dans celle de 1978 (copyrights de 1978 et 1988).

Et ce n'est que dans l'édition révisée de 1990 qu'a été opéré un changement de modèle de l'affichage de la structure des articles de ce dictionnaire, avec l'introduction de symboles diacritiques (losange éclairé et tiret) comme repères de subdivisions. Cette révision s'inspirait sur ce point du dispositif utilisé d'abord dans le $G D U$ (cf. § 5.1). L'édition refondue de 1998 adopta, elle, le troisième modèle de codification laroussien, issu du $D F C$ de 1966 (cf. $§ 5.2$ ) et caractérisé par l'introduction de symboles alphanumériques.

\subsection{Le plus petit Larousse de 1946 à 1980}

Vint ensuite Le plus petit Larousse, dictionnaire minimaliste de très petit format ${ }^{19}$ publié en 1946 sans mention de public destinataire, qui utilisait dans ses contextualisations un tiret court comme substitut du mot en adresse, mais ne recourait à aucun symbole pour la structuration des articles (figure 10) :

Figure 10 - Article dru de Le plus petit Larousse de 1946 (tirage de 1947)

$$
\text { dru, e adj. Vigoureux. Serré : pluie -. }
$$

Ce dispositif est resté inchangé dans la plus récente refonte de ce dictionnaire toujours commercialisé (depuis 2008 sous le nom de Dictionnaire micro), qui date de 1980, et dont la codification ne présente par rapport à celle de l'édition originale que des variations dans les choix affectant les caractères (et notamment la longueur du tiret anaphorique), ce que montre la figure 11 : 
Figure 11 - Article dru de Le plus petit Larousse de 1980 (tirage de 1995)

$$
\text { dru, e adj. Vigoureux. Serré : pluie - . }
$$

\subsection{Le Dictionnaire des débutants / Larousse des débutants de 1949 à 1963}

Le Dictionnaire des débutants publié en 1949 par Michel de Toro s'est également inscrit dans la lignée du NDLF de 1856 pour ses principes de codification (figure 12) :

Figure 12 - Article dru du Dictionnaire des débutants de 1949 (tirage de 1955)

dru, e adj. Serré : pluie drue, herbe drue. Vigoureux (se dit d'une personne jeune).

Ce $«$ dictionnaire manuel $»{ }^{20}$ pour enfants $«$ de huit à onze ans ${ }^{21}$, dont les articles étaient composés avec un débord de première ligne, présentait la particularité de traiter, au sein d'un même article ne constituant qu'un paragraphe, plusieurs mots d'une même famille sans recourir pour ses codifications à des moyens autres que ceux remontant au $N D L F$ de 1856, comme on peut le voir, par exemple, dans l'article dont l'adresse tête est animation (figure 13) :

Figure 13 - Article animation du Dictionnaire des débutants de 1949 (tirage de 1955)

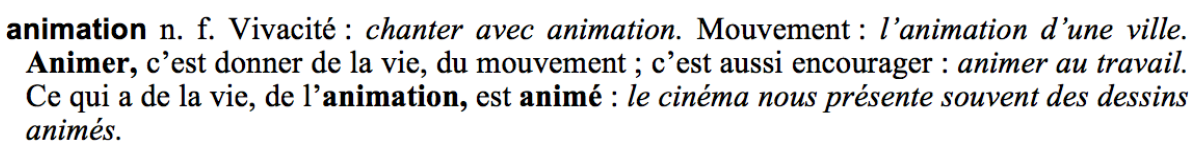

Le dispositif de mise en forme du dictionnaire est resté identique dans l'édition révisée de 1963 sous le nom de Larousse des débutants, réimprimée jusqu'au milieu des années 1980 et dont seul le texte présentait des variations (comme s.v. dru : « dru, e adj. Serré : pluie drue, herbe drue. »).

\subsection{Le Larousse de poche de 1954 à 1979}

Le dernier dictionnaire reprenant le dispositif structurant du $N D L F$ de 1856 fut, un siècle après (1954), le Larousse de poche $e^{22}$, publié par Le Livre de poche tout nouvellement créé (1953), et dépourvu de préface spécifiant des destinataires (figure 14) :

Figure 14 - Article dru du Larousse de poche de 1954 (tirage de 1955)

dru, e adj. Fort, vigoureux. Serré : pluie drue. Gaillard, vif. Adv. En grande quantité : tomber dru.

L'édition revue de 1979 garda la même codification (et le même débord de première ligne) mais connut une modification du texte relatif à l'adjectif («adj. Épais, serré, touffu. »), et c'est seulement dans l'édition refondue de 1990, lors de sa fusion avec le Petit dictionnaire français ${ }^{23}$, que, comme dans celui-ci, des symboles diacritiques (losange éclairé et tiret), issus du modèle inauguré avec le $G D U$ (cf. § 5.1), ont été introduits dans le Larousse de poche pour aider au repérage des subdivisions. En 1998, enfin, une nouvelle édition adopta, comme celle du Petit dictionnaire français, une codification inspirée de celle du $D F C$ (cf. § 5.2) qui numérotait les emplois dans les articles traitant de mots polysémiques.

\section{Postérité des évolutions du modèle de 1856}

Les évolutions du $N D L F$ qui ont été illustrées dans les figures 6 à 8 par des articles issus d'éditions de 1878 et 1886 se sont elles aussi prolongées longuement dans divers répertoires du catalogue Larousse.

\subsection{Les déclinaisons du Nouveau dictionnaire de la langue française}

Il y eut d'abord les multiples déclinaisons du $N D L F$ sous leurs diverses dénominations antérieures à 1914 (Dictionnaire complet de la langue française, Dictionnaire complet illustré, Nouveau dictionnaire illus- 
tré ${ }^{24}$, exemplifiées ici par l'article chlore du Nouveau dictionnaire illustré de 1913 reproduit dans la figure 15, qui ne différait de son prédécesseur de 1886 (cf. figure 7) que par des variations textuelles :

Figure 15 - Article chlore du Nouveau dictionnaire illustré de 1913 (247 édition)

CHLORE n. m. (gr. chlôros, jaune verdâtre). Corps simple, gazeux, d'une odeur forte et suffocante. - Gazeux ou dissous dans l'eau, le chlore, par son affinité pour l'hydrogène, détruit la partie colorante des matières végétales et animales. Aussi l'industrie l'emploie-telle pour le blanchiment des tissus. On s'en sert aussi pour détruire les miasmes. On l'utilise souvent dans les cimetières, dans les hôpitaux, dans les salles de dissection, etc.

\subsection{Le Larousse élémentaire illustré de 1914 à 1948}

À partir de 1914, le Larousse élémentaire illustré, refonte par Claude et Paul Augé des dernières versions du $N D L F^{25}$, a suivi le même modèle de codification des articles jusqu'à son édition révisée de 1948 incluse, comme l'illustre l'article chlore du millésime 1949 (figure 16) :

Figure 16 - Article chlore du Larousse élémentaire illustré de 1948 (millésime 1949)

CHLORE n. m. Corps simple (Cl), gazeux, d'une odeur forte et suffocante. - Gazeux ou dissous dans l'eau, le chlore, par son affinité pour l'hydrogène, détruit la partie colorante des matières végétales et animales. Aussi l'industrie l'emploie-t-elle pour le blanchiment des tissus. C'est un excellent désinfectant.

Dans certains articles de cette édition, l'ajout d'un composant dédié à un champ lexical signalé par un titre de rubrique en petites capitales a suscité un nouvel usage du passage à la ligne avec retrait de première ligne, sans utilisation de symbole. Le regroupement de mots opéré (surligné en gris dans la figure 17) pouvait être analogique, comme dans l'article dur (avec des subdivisions marquées par des points-virgules), ou morphologique, comme dans l'article chaleur (dans lequel sont distingués plusieurs sous-ensembles) :

Figure 17 - Articles dur et chaleur du Larousse élémentaire illustré de 1948 (millésime 1949)

DUR, E adj. Ferme, solide, difficile à entamer. Fig. Avoir l'oreille dure, entendre difficilement. Cour dur, insensible. Vie dure, austère. Paroles dures, sévères. Tête dure, lente à comprendre. Temps durs, pénibles, fâcheux. N. f. Coucher sur la dure, sur la terre nue ou sur les planches.

GROUPE D'IDÉES : consistant, ferme, solide, raide, racorni, cassant, calleux, durillon; coriace, croquant, croûte; acerbe, rude, bourru, brusque, barbare, cruel, dénaturé, inhumain, sec, froid, insensible, sévère, inflexible, rigide, tyrannique; austère, stö̈que, aguerri.

CHALEUR n. f. Qualité de ce qui est chaud. Température élevée. Fig. Ardeur : chaleur du combat.

FAMILLE DE MOTS : dérivés des rad. CHAL. (chaleureux), CHAUD. (chaudière), CHAUF (chauffer), CALÉ. (caléfaction), CALOR. (calorie), et du rad. grec THERMO. (thermomètre).

Ce n'est que dans l'édition révisée de 1955 (millésime 1956), sous le nom de Nouveau Larousse élémentaire, que des symboles diacritiques (tiret et double barre verticale) furent introduits pour matérialiser la structuration des articles par une adaptation de la codification du $G D U$ (cf. $\S 5.1$ ), et ils perdurèrent dans ce dictionnaire jusqu'aux derniers tirages de sa refonte de 1967.

\subsection{Le Petit Larousse illustré de 1905 à 1952}

Le Petit Larousse illustré, publié initialement par Claude Augé en 1905 et d'ampleur supérieure aux dictionnaires précédents ${ }^{26}$, s'est vu appliquer les principes de codification observés dans les évolutions du $N D L F$, avec la même absence foncière de symboles structurants, comme dans l'article dru (figure 18) :

Figure 18 - Article dru du Petit Larousse illustré de 1905 (millésime 1906)

DRU, E adj. (celt. drud). Fort, vigoureux. Epais, serré, touffu : blés fort drus ; pluie drue et fine. Gaillard, vif, gai : vous êtes bien dru aujourd'hui. Adv. En grande quantité, serré : tomber, semer dru. 
et l'usage du tiret pour séparer, à la fin de certains articles, des ajouts spécialisés, comme dans l'article drosère ou drosera (figure 19) :

Figure 19 - Article drosère ou drosera du Petit Larousse illustré de 1905 (millésime 1906)

DROSÈRE (zè-re) ou DROSERA (zé) n. m. Genre de droséracées carnivores, appelées aussi rossolis (rosée de soleil). - Au contact d'un insecte, la fleur s'ouvre, puis se referme en emprisonnant l'insecte, qui est tué, et digéré ensuite par le liquide acide, riche en pepsine, que sécrètent les glandes de la feuille.

Ce dispositif a été conservé jusqu'à l'édition refondue de 1952 incluse - le Nouveau Petit Larousse illustré portant le millésime 1953 -, dont l'article dru était strictement identique à celui de l'édition de 1905, tandis que l'article drosère ou drosera ne présentait par rapport à celle-ci que des variations n'affectant pas significativement sa structure : ajout de la catégorisation «n. f. » pour drosère et réécriture du texte placé après le tiret ( «- Au contact d'un insecte, la fleur se referme en l'emprisonnant, et l'insecte, tué, est digéré ensuite par le liquide acide, riche en pepsine, que sécrètent les glandes de la feuille. »).

Le principe de codification du Petit Larousse illustré a ensuite évolué en deux temps : lors de l'édition révisée de 1959, titrée Petit Larousse et millésimée 1960, fut mis en œuvre le modèle exploitant des symboles diacritiques (tiret et double barre verticale) issu du $G D U$ (cf. § 5.1); puis, à l'occasion de celle de 1988 (millésime 1989), lui succéda celui du $D F C$ (cf. § 5.2), qui a été conservé jusqu'à la refonte de 2012 incluse.

\subsection{Le Larousse classique illustré de 1910 à 1947}

Le Larousse classique illustré, publié en 1910 par Claude Augé et « destiné à répondre à toutes les exigences immédiates de l'enseignement et de l'éducation $»^{27}$, procédait du Petit Larousse illustré, dont il adaptait certains contenus en conservant la même organisation structurelle :

- l'article dru reproduisait sa source à l'identique, mise à part une option typographique différente pour l'adresse, qui n'affectait pas la structuration (figure 20):

Figure 20 - Article dru du Larousse classique illustré de 1910 ( $9^{e}$ édition, millésime 1912)

dru, e adj. (celt. drud). Fort, vigoureux. Epais, serré, touffu : blés fort drus ; pluie drue et fine. Gaillard, vif, gai : vous êtes bien dru aujourd'hui. Adv. En grande quantité, serré : tomber, semer dru.

- et il en allait de même, à une simplification textuelle près («plantes » remplaçant «droséracées » dans la définition), pour l'article drosère ou drosera, qui conservait son ajout culturel séparé du début de l'article par un tiret (figure 21):

Figure 21 - Article drosère ou drosera du Larousse classique illustré de 1910 ( $9^{\mathrm{e}}$ édition, millésime 1912)

drosère $(z \grave{e}-r e)$ ou drosera $(z e ́) \mathrm{n}$. m. Genre de plantes carnivores, appelées aussi rossolis (rosée de soleil). - Au contact d'un insecte, la fleur s'ouvre, puis se referme en emprisonnant l'insecte, qui est tué, et digéré ensuite par le liquide acide, riche en pepsine, que sécrètent les glandes de la feuille.

Les principes de mise en forme du Larousse classique illustré n'ont pas changé jusqu'à l'édition refondue de 1947 incluse, dirigée par Claude \& Paul Augé, ce dont témoigne l'article drosère ou drosera, qui différait seulement sur quelques points de celui de l'édition de 1910 : suppression de l'indication phonétique relative à drosère et réécriture du texte placé après le tiret ( «- Au contact d'un insecte, la fleur s'ouvre, puis se referme en emprisonnant l'insecte, qui est tué, et digéré ensuite par le liquide acide que sécrètent les glandes de la feuille. $»^{28}$ ).

Et ce n'est que dans le Larousse classique révisé de 1957 que des symboles diacritiques (losange noir, tiret, double barre verticale et flèche) et des dispositions de mise en page (passage à la ligne avec ou sans retrait de première ligne) ont significativement modifié l'affichage structurel des articles, plus proche dès lors de celui du $G D U$ (cf. § 5.1), et qui fut conservé jusqu'aux derniers tirages au milieu des années 1980. 


\section{Aperçus sur les deux autres modèles de codification}

Le premier modèle de structuration des articles de dictionnaires Larousse, fourni par le $N D L F$ de 1856, se caractérisait donc en particulier par le fait de ne pas utiliser significativement de symboles comme indicateurs de structuration. C'est dans des répertoires de taille et de complexité structurelle supérieures que s'est d'abord observé leur usage, avant qu'il ne se répande progressivement dans la plupart des dictionnaires du catalogue de l'éditeur. L'introduction de ces symboles s'est faite en deux temps :

- les symboles diacritiques ont été mobilisés très tôt, dès 1866, afin d'élargir la palette des codifications exploitables dans les articles d'un répertoire de référence plurivolume, le $G D U(\S 5.1)$;

- les symboles alphanumériques, eux, d'usage plus récent historiquement dans les dictionnaires (cf. n. 34), sont apparus chez Larousse dans un monovolume pédagogique, le $D F C$ de 1966 (§ 5.2).

\subsection{Le Grand dictionnaire universel du XIX ${ }^{e}$ siècle (1866-1876)}

L'échelle des articles du $G D U$, gigantesque entreprise pilotée par Pierre Larousse ${ }^{29}$, n'était pas commensurable avec celle du $N D L F$ de 1856 , comme on peut le voir à son article dru, dont la figure 22 ne reproduit que l'extrait le plus pertinent pour notre propos :

Figure 22 - Extrait de l'article dru du Grand dictionnaire universel du XIX ${ }^{e}$ siècle de 1866-1876 (t. 6, 1870)

DRU, DRUE adj. (dru, drû - v. l'étym. à la partie encycl.). Epais, touffu, serré : Une herbe DRUE. Une pluie DRUE et menue. (Acad.)

- Fort, vigoureux, en parlant des petits oiseaux qui sont prêts à s'envoler du nid : Ces moineaux sont DRUS ; ils sont DRUS comme père et mère. (Acad.)

- Fam. Gaillard, vif, gai, décidé : Vous voilà bien DRU aujourd'hui. (Acad.) Je trouvai à l'hôtel de Sully $M^{\text {le }}$ de Hannoy, mariée au petit-fils du vieux comte de Montrevel; jamais vous n'avez vu une mariée si DRUE ; elle va droit à son ménage et dit déjà : mon mari. ( $\mathrm{M}^{\mathrm{me}}$ de Sév.) Nous ne sommes plus gaillards et DRUS d'humeur comme l'était un Vivonne au temps de Louis XIV. (Ste-Beuve.)

... Malgré moi, l'on m'a jointe avec vous ;

Vous, vieux penard ; moi, fille jeune et drue.

\section{LA FonTAINE.}

- Adv. D'une manière serrée; en grande quantité : Arbres plantés DRU. Ces blés poussent DRU. Qui sème $\mathrm{DRU}$, dit un ancien proverbe, récolte clair, et qui sème clair récolte $\mathrm{DRU}$. (Raspail.) On appelle semer en pépinière l'action de semer très-DRU, et dans un coin du champ, des plantes que l'on se propose de repiquer ensuite. (Raspail.)

Sire, dit-il, le nœud du mariage,

Damne aussi $d r u$ qu'aucuns autres états.

LA FonTAINE.

- A coups redoublés, précipités : II frappe fort et DRU.

- Jaser, caqueter dru, Parler beaucoup et vite :

Caquet Bon-Bec alors de jaser au plus dru. LA FONTAINE.

De telles gens il est beaucoup

Qui prendraient Vaugirard pour Rome,

Et qui, caquetant au plus dru,

Parlent de tout et n'ont rien vu.

LA Fontaine.

- s. f. Ornith. Un des noms vulgaires du proyer.

— Encycl. Linguist. [développement sur l'étymologie et l'histoire de l'adjectif $d r u$ ]

Du point de vue de son contenu informationnel, cet article se distinguait de celui du NDLF, d'une part, par le plus large spectre des données linguistiques décrites (distinction de deux sens principaux pour l'adverbe, ajout des expressions jaser dru et caqueter dru et d'un emploi comme substantif féminin), et, d'autre part, par des composants supplémentaires (une rubrique étymologique, des citations dictionnairiques 
(«(Acad.) ») ou littéraires avec indication de leur auteur - dans le texte pour celles en prose, sorties pour celles en vers -, et un développement encyclopédique, en l'occurrence articulé à la rubrique étymologique initiale, et presque deux fois plus long, en nombre de lignes, que la première partie de l'article).

Pour l'affichage des composants et des subdivisions de la microstructure, tous les types de moyens étaient utilisés, à l'exception des symboles alphanumériques. Aux procédés déjà mis en œuvre dans le $N D L F$ (cf. figure 1) se sont ajoutés

- d'une part un usage plus important des moyens typographiques, avec

- un corps plus petit pour les caractères des citations sorties et des noms de leurs auteurs ;

- la mise en relief du mot ou de l'expression illustré(e) : dans les contextualisations forgées et dans les citations non sorties, par de petites capitales en romain dans un contexte en italique, et, dans les citations sorties, par de l'italique dans un contexte en romain ;

- corrélativement, un traitement contextuellement contrastif du nom des auteurs de textes cités : par des caractères romains normaux pour les citations non sorties et par des petites capitales en romain pour les citations sorties ;

- et une exploitation importante des moyens de la mise en page : passage à la ligne avec retrait de première ligne pour chaque subdivision de l'article, qu'elle soit grammaticale, sémantique, lexicale ou encyclopédique, passage à la ligne avec un centrage relatif du texte pour les citations sorties, et passage à la ligne et décalage vers la droite pour les noms de leurs auteurs ;

- et d'autre part le recours à des symboles diacritiques pour matérialiser les limites de subdivisions, qui pouvaient être

- des tirets longs en renforcement de chaque passage à la ligne avec retrait de première ligne,

- et, pour des subdivisions subordonnées à celles introduites par ces tirets, des doubles barres verticales à l'intérieur de certains paragraphes, comme dans l'article drôlerie (figure 23) :

Figure 23 - Extrait de l'article drôlerie du Grand dictionnaire universel du XIX siècle de 1866-1876 (t. 6, 1870)

DRÔLERIE s. f. (drô-le-rî — rad. drôle). Caractère de ce qui est drôle : C'est une scène d'une DRÔLERIE incroyable.

- Action ou parole drôle ; trait de gaillardise, de bouffonnerie : Voilà une plaisante DRÔLERIE. Faire, dire des DRÔLERIES. Qu'est-ce? Me ferez-vous voir votre petite DRÔLERIE? (Mol.) \|| Acte de malice, tour malin: C'est une mauvaise plaisanterie, une DRÔLERIE échappée des mains d'un oisif qui veut s'amuser à mes dépens. (L. Gozlan.)

Dans l'ensemble des procédés qui singularisent la mise en forme des articles du $G D U$ par rapport au modèle du $N D L F$, le plus significatif, en matière de structuration, est la conjonction du passage à la ligne, du retrait de première ligne et du tiret long pour séparer les subdivisions majeures.

Ce modèle structurel alliant d'une part les codifications retenues ci-dessus comme le différenciant du premier modèle et d'autre part les doubles barres verticales et des moyens graphématiques (notamment des points-virgules) pour les subdivisions subalternes s'est propagé, sous une forme similaire ou apparentée, dans les grands dictionnaires encyclopédiques plurivolumes qui lui ont succédé jusqu'au deuxième tiers $\mathrm{du} \mathrm{XX}^{\mathrm{e}}$ siècle, et des versions plus ramassées de ce dispositif ont été mises en œuvre dans divers dictionnaires ultérieurs de dimensions et d'ambitions plus réduites, dont certains ont été évoqués aux $\S \S 3$ et 4.

À partir des années 1950, toutefois, une complexification de l'emploi de symboles diacritiques est venue suppléer, partiellement ou totalement selon les ouvrages, les effets de mise en page dans la codification de la structure des articles d'un large ensemble de dictionnaires en un ou plusieurs volumes. 


\subsection{Le Dictionnaire du français contemporain de 1966}

Les traitements fournis dans le $D F C$ de 1966 pour chaque unité linguistique étaient souvent commensurables à ceux du NDLF de 1856. Toutefois, sa vocation étant de « répondre aux nécessités nouvelles de l'enseignement moderne $d u$ français $»^{30}$, ceux relatifs à des items morphosémantiquement apparentés et partageant des propriétés syntaxiques étaient regroupés au sein de blocs d'articles ${ }^{31}$ qui déstructuraient l'ordre alphabétique (les préfixés étant traités à la suite de leurs bases), tandis que des items homographes impliqués dans des regroupements lexicaux différents donnaient lieu à une démultiplication d'articles. Cette double macrostructure impliquait un bon repérage des articles au sein des blocs qui les regroupaient dans un même paragraphe, ce qui se faisait au moyen d'un losange noir précédant chaque adresse subordonnée. Ce signe diacritique ayant un rôle de repère macrostructurel, c'est aux symboles alphanumériques que revint la codification de la distinction des emplois dans les articles traitant de mots polysémiques ${ }^{32}$.

Dans son article dru, reproduit en figure 24, le $D F C$, composé au carré avec, comme dans différents répertoires précédemment évoqués, des caractères à empattement sauf pour les adresses principales (en caractères bâton), proposait des développements un peu plus réduits que ceux du NDLF (cf. figure 1), centrés sur les emplois contemporains ${ }^{33}$ (ce qui excluait le sens non figuré de l'adjectif) et limités, pour l'adverbe, à la présentation de contextualisations, et sa symbolique ne mobilisait que le losange noir séparant les traitements de l'adjectif et de l'adverbe.

Figure 24 - Article dru du Dictionnaire du français contemporain de 1966

dru, e [dry] adj. Se dit de ce qui est serré, épais : Une barbe drue. Des cheveux drus. $\bullet$ adv. : La pluie, les balles tombent dru. L'herbe pousse dru.

Au sein d'un bloc, le losange noir séparait aussi bien les traitements d'items homographes catégorisés différemment que ceux de dérivés, ce qui s'observe, en figure 25 , dans un bloc qui fait précéder son adresse principale par un ordonnateur numérique. La valeur de ce chiffre arabe, en police bâton comme l'adresse qu'il précède, reflète l'ordre des développements consacrés aux noms féminins fable traités comme deux homonymes et donnant lieu à ce titre à des articles distincts du fait de leur implication dans des familles lexicales différentes : (i) 1. fable n. f., fablier n. m., fabliau n. m. et fabuliste n. m., et (ii) 2. fable n. f., fabulation n. f., fabulateur, trice adj. et n., fabuleux, euse adj., fabuleusement adv. et fabuler v. intr.

Cet article 2. fable montre aussi que d'autres ordonnateurs numériques ont été exploités, dans le traitement du nom fable considéré et de l'adjectif fabuleux, pour introduire chacune des subdivisions linguistiques majeures, séparées en outre par un tiret long.

Figure 25 - Article 2. fable du Dictionnaire du français contemporain de 1966

2. fable [fabl] n. f. $1^{\circ}$ Récit mensonger (langue soutenue) : Vous nous racontez des fables (syn. : HISTOIRES ; fam. : BONIMENTS ; contr. : VÉRITÉ). Il ne sait plus quelle fable inventer. $-2^{\circ}$ Sujet des conversations et de la risée publiques: Il est devenu la fable du quartier. - fabulation n. f. Fait de substituer un récit imaginaire à la réalité vécue : Cet enfant a le goût de la fabulation (syn. : MYTHOMANIE). — fabulateur, trice adj. et n. : Quelle faculté fabulatrice! - fabuleux, euse adj. $1^{\circ}$ Du domaine de l'imagination (littér.) : Un personnage fabuleux (syn. : IMAGINAIRE, CHIMÉRIQUE). Un animal fabuleux (syn. : LÉGENDAIRE). $2^{\circ}$ Qui dépasse l'imagination: Amasser une fortune fabuleuse (syn.: FANTASTIQUE, FORMIDABLE, INOUİ). — fabuleusement adv. Au sens 2 de l'adj. : Etre fabuleusement riche (syn. : FORMIDABLEMENT). $\bullet$ fabuler v. intr. Faire un récit imaginaire ; exagérer.

L'innovation signalétique observée dans le $D F C$ est à corréler à la richesse des descriptions linguistiques qui y étaient développées, qu'elles soient morphologiques (par les regroupements d'articles), combinatoires (en particulier pour les unités linguistiques qui en régissent d'autres), sémantiques (par l'homonymisation poussée des traitements comme par le réseau des synonymes et contraires associés aux descriptions des emplois) ou énonciatives (notamment par le marquage des registres).

Jean Dubois, maître d'œuvre du DFC et responsable des dictionnaires de langue chez Larousse dans les années 1960-1980, a nécessairement dû jouer ensuite un rôle dans la propagation du modèle structurel du DFC (refondu en 1980 avec le même dispositif sous le nom de Dictionnaire du français contemporain illustré) à divers dictionnaires présentant des descriptions linguistiques élaborées selon des principes pa- 
rents (au premier rang desquels le Lexis. Dictionnaire de la langue française de 1975, toujours commercialisé). Pour autant, Larousse est loin d'avoir été pionnier en matière d'utilisation d'ordonnateurs alphanumériques dans les articles de dictionnaires monolingues, cette pratique, déjà bien illustrée au XIX ${ }^{\mathrm{e}}$ siè$\mathrm{cle}^{34}$, ayant d'abord été réactivée au $\mathrm{XX}^{\mathrm{e}}$ dans le Dictionnaire alphabétique et analogique de la langue française de Paul Robert (1953-1964), après un demi-siècle de "vide lexicographique »" ${ }^{35}$. L'introduction de symboles alphanumériques dans le $D F C$ s'est donc inscrite en fait dans une évolution générale dont il est un des premiers jalons, qui s'est aujourd'hui étendue à toute la lexicographie française, mais dans laquelle Larousse n'a pas été précurseur, puisque ce n'est qu'en 1988 que ce principe de codification a été appliqué au Petit Larousse illustré (millésime 1989).

\section{Conclusion}

Depuis 160 ans que Larousse produit des dictionnaires, une esthétique d'économie, une sorte de "ligne claire", semble régir leur mise en forme, par delà les différences induites par celles des contenus des répertoires et les évolutions liées aux techniques et aux modes. Nous sommes si habitués à pratiquer des dictionnaires dont les articles sont structurés par des chiffres (souvent) et des lettres (parfois) qu'il y a lieu d'être surpris en découvrant que cela ne fait qu'un quart de siècle qu'ils ont investi le Petit Larousse. À la traîne, Larousse ? Non : fonctionnel et sobre. Prudent aussi, peut-être : numéroter les sens des mots, n'estce pas déjà suggérer que le sens 1 est premier par rapport au sens 2 ? On peut faire beaucoup de choses avec de la typographie, des majuscules et de la ponctuation, alors à quoi bon surcharger la mise en forme du $N D L F$ et des petits utilitaires généralistes ou didactiques postérieurs ? Les besoins des grands dictionnaires sont évidemment autres, et les moyens s'adaptent, mais à nouveau sans excès, avec l'usage récurrent du passage à la ligne et du tiret polyvalent ${ }^{36}$ dans le $G D U$ et ensuite. Bien sûr, il faut suivre l'évolution collective, observer les pratiques concurrentes, ne pas paraître dépassé, mais sans perdre son âme on dirait aujourd'hui son ADN d'entreprise. Quand le Petit Larousse intégra les symboles alphanumériques en 1988, il s'agissait encore d'une évolution fonctionnelle, puisque l'organisation des articles avait évolué vers un plan plus sémantique, à la manière des dictionnaires Robert. Et puis la position de leader durable sur un marché conforte le parti-pris de sobriété : libre aux challengers de se faire remarquer.

\section{Références bibliographiques}

\section{Dictionnaires}

DFC = Dictionnaire du français contemporain.

Dictionnaire alphabétique et analogique de la langue française. Les mots et les associations d'idées. [Robert, P.]. 6 vol. Paris : Société du Nouveau Littré. 1953-1964.

Dictionnaire de la langue française. [Littré, É.]. 4 vol. Paris : Librairie Hachette et $C^{\text {ie }} .1863-1873$.

Dictionnaire des débutants. [de Toro, M.]. Paris : Librairie Larousse. 1949. - Larousse des débutants. [de Toro, M.]. Nouv. éd. 1963. Dernier tir. connu : 1985.

Dictionnaire du français contemporain. [Dubois, J., Lagane, R., Niobey, G., Casalis, D., Casalis, J. \& Meschonnic, H.]. Paris : Librairie Larousse. 1966. - Dictionnaire du français contemporain illustré. Nouv. éd. 1980.

Dictionnaire général de la langue française du commencement du XVII siècle jusqu'à nos jours. [Hatzfeld, A., Darmesteter, A. \& Thomas, A.]. 2 vol. Paris : Librairie Delagrave. 1890-1900.

Dictionnaire micro : voir Le plus petit Larousse.

$G D U=$ Grand dictionnaire universel $d u X I X^{e}$ siècle.

Grand dictionnaire universel du XIX $X^{e}$ siècle [...]. [Larousse, P.]. 15 vol. Paris : Administration du Grand Dictionnaire Universel. 1866-1876. 
Larousse classique illustré. Nouveau dictionnaire encyclopédique. [Augé, C.]. Paris : Librairie Larousse. 1910. [Augé, C. \& Augé, P. (dir.)]. Nouv. éd. entièrement refondue. 1947. - Larousse classique. Dictionnaire encyclopédique. Nouv. éd. 1957. Dernier tir. connu : 1985.

Larousse de poche. Paris : Librairie Larousse / Le Livre de poche. 1954. - Nouv. éd. revue et mise à jour. 1979. Nouv. éd. augmentée fusionnée avec le Petit dictionnaire français. Larousse / Le Livre de poche. 1990. - Éd. « entièrement nouvelle». Larousse. 1998.

Larousse des débutants : voir Dictionnaire des débutants.

Larousse élémentaire illustré. [Augé, C. \& Augé, P. (dir.)]. Éd. refondue et augmentée. Paris : Librairie Larousse. 1914. - [Augé, C. \& Augé, P. (dir.)]. Éd. entièrement refondue. Millésime 1949. 1948. - Nouveau Larousse élémentaire. Nouv. éd. Millésime 1956. 1955. - Nouv. éd. 1967. Dernier tir. connu : 1978.

Le plus petit Larousse. Paris : Librairie Larousse. 1946. - Nouv. éd. 1980. - Tir. actuels (depuis 2008) : Dictionnaire micro. Tir. le plus récent : 2012.

Lexis. Dictionnaire de la langue française. Paris : Librairie Larousse. 1975. - Le Lexis. Le dictionnaire érudit de la langue française. Nouv. éd. Larousse. 2009.

$N D L F=$ Nouveau dictionnaire de la langue française.

Nouveau dictionnaire de la langue française. [Larousse, P.]. $3^{\mathrm{e}}$ éd. Paris : Larousse et Boyer Libraires-Éditeurs. 1856. - [Larousse, P.]. 43 éd. « augmentée d'une partie toute nouvelle». Aug. Boyer \& C ${ }^{\mathrm{ie}}$, Libraires-Éditeurs. 1878. - [Larousse, P.]. $66^{\mathrm{e}}$ éd. « illustrée et considérablement augmentée ». Librairies Larousse \& Boyer réunies, $\mathrm{V}^{\mathrm{ve}}$ P. Larousse \& $\mathrm{C}^{\mathrm{ie}}$, Imprimeurs-Éditeurs. 1886. - Nouveau dictionnaire illustré. [Larousse, P.]. $247^{\mathrm{e}}$ éd. Librairie Larousse. 1913.

Nouveau dictionnaire illustré : voir Nouveau dictionnaire de la langue française.

Nouveau Larousse élémentaire : voir Larousse élémentaire illustré.

Nouveau Petit Larousse illustré : voir Petit Larousse illustré.

Petit dictionnaire français. [Augé, P.]. 1936. Paris : Librairie Larousse. - Nouv. éd. [Augé, P. (dir.)]. 1956. - Éd. refondue. 1978. - Éd. actualisée. 1988. - Nouv. éd. fusionnée avec le Larousse de poche. Larousse. 1990. - Éd. « entièrement nouvelle ». 1998.

Petit Larousse : voir Petit Larousse illustré.

Petit Larousse illustré. Nouveau dictionnaire encyclopédique. [Augé, C. (dir.)]. Millésime 1906. Paris : Librairie Larousse. 1905. - Nouveau Petit Larousse illustré. Dictionnaire encyclopédique. [Augé, C. \& Augé, P.]. Éd. du centenaire refondue et augmenté. Millésime 1953. 1952. - Petit Larousse. Nouv. éd. Millésime 1960. 1959. Petit Larousse illustré. Nouv. éd. Millésime 1989. 1988. - Le Petit Larousse illustré. Nouv. éd. Millésime 2012. Larousse. 2011.

\section{Autres références}

Boulanger, J.-C. (2003). Les inventeurs de dictionnaires. De l'eduba des scribes mésopotamiens au scriptorium des moines médiévaux. Coll. Regards sur la traduction. Ottawa : Les Presses de l'Université d'Ottawa.

Brandon, E.E. (1904). Robert Estienne et le dictionnaire français au XVI siècle. Baltimore : J.H. Furst. Réimpr. Genève : Slatkine Reprints. 1967.

Bray, L. (1989). Consultabilité et lisibilité du dictionnaire : aspects formels. In F.J. Hausmann, O. Reichmann, H.E. Wiegand \& L. Zgusta (Hrsg.). T. 1, art. 15, 135-146.

Bray, L. (1990). La lexicographie française des origines à Littré. In F.J. Hausmann, O. Reichmann, H.E. Wiegand \& L. Zgusta (Hrsg.). T. 2, art. 185, 1788-1818.

Buridant, C. (1986). Lexicographie et glossographie médiévales. Esquisse de bilan et perspectives de recherche. Lexique, 4, 9-46.

Corbin, F. \& Corbin, P. (2008). Les dictionnaires sont des voitures comme les autres. In F. Maniez \& P. Dury (éds). Lexicographie et terminologie : histoire de mots. Hommage à Henri Béjoint. Gap : Louis Jean Imprimeur. 47-65. 
Corbin, P. (1985). Le monde étrange des dictionnaires (6). Le commerce des mots. Lexique, 3, 65-124.

Corbin, P. (1989a). Lire les dictionnaires. Pour la constitution en champ de recherches de la lecture critique des textes lexicographiques français contemporains, Le français dans le monde. Recherches et applications, numéro spécial (« Lexiques »), 31-41

Corbin, P. (1989b). Pour une lecture généalogique des dictionnaires généraux monolingues français contemporains. In G. James (ed.), Lexicographers and their Works. Coll. Exeter Linguistic Studies, vol. 14. University of Exeter. 29-54.

Corbin, P. (1995). Le monde étrange des dictionnaires (8). Du Petit Robert (1967) au Micro Robert (1971) : le recyclage de citations. Lexique, 12-13, 125-145.

Corbin, P. \& Gasiglia, N. (2012). Structuration formelle et structuration conceptuelle des articles des dictionnaires Larousse : repères pour une approche historique. Communication au $5^{\mathrm{e}}$ colloque Pierre Larousse, «Larousse : lexicographie et pédagogie en héritage », Toucy, 11-12 mai 2012.

Corbin, P. \& Gasiglia, N. (2013). Structurations formelle et conceptuelle des articles de dictionnaires : comparaison de codifications employées chez Larousse en 1856 et 1997. Communication au XXVII ${ }^{\mathrm{e}}$ Congrès international de linguistique et de philologie romanes, Nancy, 15-20 juillet 2013. À paraître dans les actes.

Gaudin, F. \& Guespin, L. (2000). Initiation à la lexicologie française. De la néologie aux dictionnaires. Coll. Champs linguistiques. Manuels. Bruxelles : Duculot.

Hausmann, F.J. (1989). Dictionary criminality. In F.J. Hausmann, O. Reichmann, H.E. Wiegand \& L. Zgusta (Hrsg.). T. 1 , art. $11,97-101$

Hausmann, F.J., Reichmann, O., Wiegand, H.E. \& Zgusta, L. Hrsg. (1989-1991). Wörterbücher / Dictionaries / Dictionnaires. Ein internationales Handbuch zur Lexikographie / An International Encyclopedia of Lexicography / Encyclopédie internationale de lexicographie. 3 vol. Berlin / New York : Walter de Gruyter.

Hausmann, F.J. \& Wiegand, H.E. (1989). Component parts and structures of general monolingual dictionaries: a survey. In F.J. Hausmann, O. Reichmann, H.E. Wiegand \& L. Zgusta (Hrsg.). T. 1, art. 36, 328-360.

Martinez, C. (2012). L'orthographe des dictionnaires français. La construction de la norme graphique par les lexicographes. Coll. Lexica. Mots et dictionnaires, vol. 22. Paris : Honoré Champion Éditeur.

Martinez, C. (2013). La comparaison de dictionnaires comme méthode d'investigation lexicographique. Lexique, 21, 193-220.

Matoré, G. (1968). Histoire des dictionnaires français. Paris : Librairie Larousse.

Mollier, J.-Y. \& Dubot, B. (2012). Histoire de la librairie Larousse (1852-2010). Paris : Fayard.

Morvan, D. (2009). De l'articulation du discours lexicographique : la fonction démarcative de la "grande division". Cahiers du dictionnaire, 1, 53-67.

Quemada, B. (1968). Les dictionnaires du français moderne 1539-1863. Étude sur leur histoire, leurs types et leurs méthodes. Paris / Bruxelles / Montréal : Didier.

Rey, A. (1988). À propos d'un "vide lexicographique" et de l'encyclopédisme dans les dictionnaires français entre le Dictionnaire général et le Robert (1900-1960). Travaux de linguistique et de philologie, XXVI, 191-204.

Rey, A. (1990). La lexicographie française depuis Littré. In F.J. Hausmann, O. Reichmann, H.E. Wiegand \& L. Zgusta (Hrsg.). T. 2, art. 186, 1818-1843.

Wagner, R.-L. (1967). Les vocabulaires français. I. Définitions - Les dictionnaires. Coll. Orientations. Paris / Bruxelles / Montréal : Didier.

Weijers, O. (1991). Dictionnaires et répertoires au moyen âge. Une étude du vocabulaire. Coll. Études sur le vocabulaire intellectuel du moyen âge, vol. IV. Turnhout : Brepols.

Wooldridge, T.R. (1977). Les débuts de la lexicographie française. Estienne, Nicot et le "Thresor de la langue francoyse", 1606. Toronto : University of Toronto Press.

Zgusta, L. (1986). Eine Kontroverse zwischen der deutschen und der englischen Sanskrit-Lexikographie: Erster Schritt zu einer Theorie des Abschreibens. In A. Schöne (Hrsg.). Kontroversen, alte und neue. Akten des VII. internationalen Germanistische-Kongresses, Göttingen, 1985. Tübingen : Max Niemeyer Verlag. Band 3, 248-252. 
${ }^{1}$ Cf. Wagner (1967 : 99-108), Matoré (1968 : 49-52), Buridant (1986), Bray (1990 : 1788-1790), Weijers (1991), Gaudin \& Guespin (2000 : 16-18), Boulanger (2003, chap. 7-10).

${ }^{2}$ Cf. Brandon (1904), Wagner (1967 : 91, 106-107, 121, 123), Quemada (1968 : 12, 39-40, 45-46, 63, 160), Matoré (1968: 58-60), Gaudin \& Guespin (2000:18-25).

${ }^{3}$ Cf. Hausmann (1989 : 99).

${ }^{4}$ Cf. Zgusta (1986), Rey (1990 : 1825).

${ }^{5}$ Cf. Corbin (1985), Corbin \& Corbin (2008, $\S \S 3$ et 4).

${ }^{6} \mathrm{Cf}$. Corbin (1989a : 36).

${ }^{7}$ Cf., exemplairement, Wooldridge (1977).

${ }^{8}$ Cf. Martinez (2012, 2013).

${ }^{9}$ Cf. Corbin (1989b).

${ }^{10}$ Cf. Corbin (1995).

${ }^{11}$ Bray (1989).

${ }^{12}$ Cf. Corbin \& Gasiglia (2012).

${ }^{13}$ Cf. Corbin \& Gasiglia (2013).

${ }^{14}$ Il serait pertinent d'apprécier les options de codification des premiers dictionnaires Larousse par rapport à celles en usage chez d'autres éditeurs lors de leur parution, mais ce serait l'objet d'une autre étude.

${ }^{15}$ XIV +714 p. de $11 \times 16,5 \mathrm{~cm}$. Sur la nouveauté et l'intérêt commercial du format de ce dictionnaire, cf. Mollier \& Dubot (2012:76-78).

${ }^{16} \mathrm{Ce}$ sont ceux de la Bibliothèque Nationale de France qui ont été numérisés et sont accessibles depuis le site gallica.bnf.fr.

${ }^{17}$ Pp. 635-700 dans l'édition de 1856 ; pp. 1-58, avec une numérotation autonome, dans celle de 1878.

${ }^{18} 820$ p. de $10 \times 13,5 \mathrm{~cm}$.

19584 p. de $5,5 \times 8 \mathrm{~cm}$.

${ }^{20}$ «Aux lecteurs », deuxième page. Il comportait [8] +616 p. de $10,5 \times 16 \mathrm{~cm}$ et sa page de titre faisait état de «18.000 MOTS DÉFINIS ».

${ }^{21}$ «Aux lecteurs », première page.

${ }^{22}[4]+506$ p. de $11 \times 16,5 \mathrm{~cm}$.

${ }^{23}$ À l'issue de leur refonte de 1990, malgré l'unification de leur texte et leur composition commune avec un débord de première ligne et sans passages à la ligne, le Petit dictionnaire français et le Larousse de poche sont restés différenciés par leur typographie : caractères bâton pour le premier, à empattement pour le second (sauf l'adresse).

${ }^{24}$ Détailler l'histoire compliquée de cette filière éditoriale constituerait en soi l'objet d'une autre publication.

${ }^{25}$ « Aux lecteurs », première page. Il comportait alors [8] + 1275 p. de 10,5 × 15,5 cm.

${ }^{26}[8]+1664$ p. de $13,5 \times 19 \mathrm{~cm}$.

${ }^{27}$ « Aux lecteurs », première page. Il comportait [8] + 1100 p. de $13 \times 19 \mathrm{~cm}$.

${ }^{28}$ Consultation effectuée dans le millésime 1952 (1951).

${ }^{29} 15$ vol. non illustrés de $23 \times 31 \mathrm{~cm}$, composés sur quatre colonnes serrées.

${ }^{30}$ « Avant-propos », p. V. Il comportait XXIV + 1226 p. de $17 \times 22,5 \mathrm{~cm}$.

${ }^{31}$ Des « nids » dans la terminologie de Hausmann \& Wiegand (1989 : 336). 
${ }^{32}$ Dans le $D F C$ comme dans d'autres dictionnaires, les chiffres arabes suffisaient le plus souvent à la numérotation des unités de traitement dans les articles, chiffres romains et/ou lettres (spécialement majuscules) ne servant que pour des mots linguistiquement complexes et pouvant alors être assortis de passages à la ligne (par exemple s.v. 1. faire v. tr.).

33 « Le Dictionnaire du français contemporain vise à présenter un état actuel du lexique usuel. » (« Avant-propos », p. III). L'ordre de grandeur de sa nomenclature était évalué à « environ 25000 » mots (ibid.).

${ }^{34}$ En particulier dans le Dictionnaire de la langue française d'Émile Littré (1863-1873) et dans le Dictionnaire général de la langue française d'Adolphe Hatzfeld, Arsène Darmesteter \& AntoineThomas (1890-1900).

${ }^{35}$ Cf. Rey (1988), qui caractérise ainsi la lexicographie de la première moitié du XX ${ }^{\mathrm{e}}$ siècle, dominée par les dictionnaires "encyclopédiques" et déficitaire en dictionnaires "de langue".

${ }^{36}$ Cf. Morvan (2009). 